\title{
Iliopsoas Metastasis from Cervix Carcinoma Masquerading as Psoas Abscess
}

\author{
Moufid Kamal $^{*}$, Driss Touiti, Hassan Jouhadi and Abdeltif Benider
}

Military Hospital Avicenne, Marrakech, Morocco

\begin{abstract}
Objectives: Contribution of a case of unusual spread of cervical carcinoma mimicking iliopsoas abscess fifteen years after initial radiotherapy.
\end{abstract}

Method: A 65-year-old white woman with history of squamous cell carcinoma of uterine cervix treated 15 years ago, was referred to our department with clinical and imaging's findings simulating a psoas abcess.

Result: Colposcopy and punch biopsy confirmed the diagnosis of carcinoma of the cervix. Needle aspirate of cystic mass yielded a watery chocolate-colored. Cytopathology revealed cells suggestive of squamous cell carcinoma consistent with the diagnosis of metastasis from carcinoma cervix. Consultations with radiation therapy and medical oncology suggested no additional therapy. The patient became increasingly cachectic and died with generalized metastasis 3 months after admission.

Conclusion: Through this case, the authors highlights the fact that metastasis from squamous cell carcinoma of the cervix can present as a psoas abscess and demonstrates certain difficulties in diagnosis even with the newer imaging modalities.

Keywords: Metastasis; Iliopsoas muscle; Abscess; Cervix carcinoma; Cytopathology

Abbreviations: CT-scan: Computed-tomography Scan; MRI: Magnetic Resonance Imaging; IPM: Iliopsoas Metastasis

\section{Introduction}

The iliopsoas compartment may be affected by several diseases, including infections, tumors and hemorrhages. Isolated metastases to iliopsoas muscle are uncommon and may be misdiagnosed when it is the presenting symptom, especially in the absence of a known primary tumor [1]. Radiologically and clinically these lesions can be mistaken for abscesses [2,3]. We describe a rare case of secondary psoas abscess arising from an advanced squamous cell carcinoma of the cervix fifteen years after initial radiotherapy.

\section{Case Presentation}

A 65 years-old women was referred to our department with a complaint of chronic backache which had progressively worsened over a period of 3 months. This was associated with a three-day history of fever and chills and a painful left hip. She also claimed to have lost a significant amount of weight over the last two months. She was febrile, drowsy and poorly oriented. Physical examination showed left hip maintained in a flexed position while abdominal examination revealed a vague, tender, fluctuant mass over the left iliac fossa. She had been diagnosed as a case of squamous cell carcinoma of the cervix, stage III and had completed radiotherapy fifteen years previously. A speculum examination revealed an ulcerative tumor which had obliterated the entire cervix. Colposcopy and punch biopsy confirmed the diagnosis of carcinoma of the cervix. Laboratory findings on admission: urinalysis was normal, haemoglobin level of 8,4 g, C-reactive protein 40-200 $\mathrm{mg} / \mathrm{L}$ and total white count of $2000 / \mathrm{mm}^{3}$ with $88 \%$ polymorph. A computed tomography (CT) scan revealed a large septated left psoas abscess which was pointing at the level of the groin associated with bone involvement of the lumbar vertebrae (Figure 1). Intravenous broad-spectrum antibiotics with anaerobic and gram-negative coverage (third cephalosporins aminoglycosides) were started and it was decided to attempt percutaneous drainage of the abscess. An 18-gauge 20-cm-long sheathed needle (Boston Scientific/Medi-Tech, Rungis, France) was inserted into the most distal portion of the fluid collection. Initially, a diagnosis of spinal tuberculosis with right psoas abscess was made due to the endemicity of tuberculosis in this part of the world. Needle aspirate of cystic mass yielded a watery chocolate-

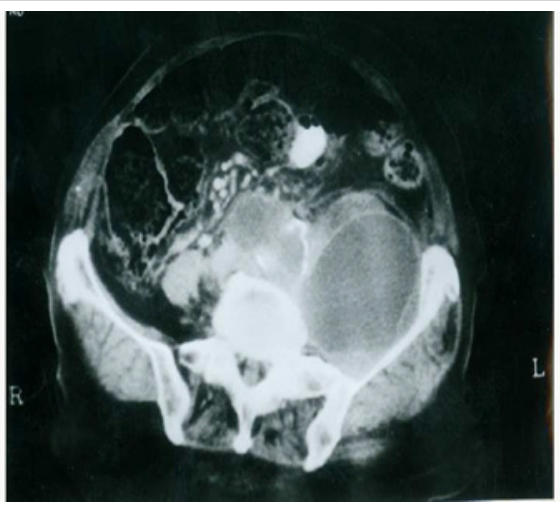

Figure 1: Contrast-enhanced CT shows septated cystic metastases in left iliopsoas muscle associated with bone involvement of the lumbar vertebrae.

*Corresponding author: Moufid Kamal, Military Hospital Avicenne, Marrakech 40000, Morocco, Tel: 00212661191099; Fax: 00212522273838; E-mail: monsieurmoufid@yahoo.fr

Received March 05, 2012; Accepted April 11, 2012; Published April 13, 2012

Citation: Kamal M, Touiti D, Jouhadi H, Benider A (2012) Iliopsoas Metastasis from Cervix Carcinoma Masquerading as Psoas Abscess. J Cancer Sci Ther 4 075-076. doi:10.4172/1948-5956.1000114

Copyright: $\odot 2012$ Kamal M, et al. This is an open-access article distributed under the terms of the Creative Commons Attribution License, which permits unrestricted use, distribution, and reproduction in any medium, provided the original author and source are credited. 
colored. The aspirate was sent for aerobic, fungal and mycobacterium tuberculosis cultures but all were negative. Cytopathology revealed cells suggestive of squamous cell carcinoma consistent with the diagnosis of necrotic metastatic squamous cell carcinoma. The poor physical state of this patient and the presence of metastases contraindicated surgical treatment and made this option unacceptable. Consultations with radiation therapy and medical oncology suggested no additional therapy. The patient became increasingly cachectic and died with generalized metastasis 3 months after admission.

\section{Discussion}

Skeletal muscle represents approximately $50 \%$ of total body mass. However, metastases into the iliopsoas muscle are a rare phenomenon. Although the reasons for its rarity are not established, some factors that might account for the infrequency of skeletal muscle metastasis have been postulated such as dislodgment of tumor cells by blood turbulence and muscle contraction, unfavorable $\mathrm{pH}$ and effect of protease inhibitors within the muscle unfavorable to the growth of malignant tissue [1-4].

Several metastatic spread have been postulated in the literature. According to Yang et al. [2] modes of involvement with malignant disease of the iliopsoas include primary tumors (20\%) direct invasion from adjacent nodes (55\%), local recurrence (8\%), metastatic deposits adjacent to the vertebrae (12\%) and hematogenous (extremely rare). The low incidence reported can be explained by the fact that only a fraction of patients survive long enough for clinical detection while skeletal muscle metastasis occurs as a late event in progression of disease. However, carcinoma of the cervix spreads locally and metastasizes late [5].

To complement the clinical assessment and provide further characterization of IPM, CT and MRI has been commonly used. IPM has a broad spectrum of appearances. Contrast enhanced CT is useful in the detection of abscess and neoplasm. Intramuscular rimenhancing lesions with central hypo attenuation were typically seen in these cases [6]. Physicians must be aware of the associated radiologic features such as the presence of adenopathy, bone destruction and discontinuity of fascial planes. The recent study by Surov et al. [4] of 5,170 patients with metastasized cancer identified on computed tomography showed muscle metastases in 61 patients (80 lesions). Among them, iliopsoas muscle was involved in $27.5 \%$.The investigators reported five types of lesion: intramuscular mass (type I), abscess-like intramuscular lesion (type II), diffuse metastatic muscle infiltration (type III), multiple muscle calcification (type IV) and intramuscular bleeding (type V). In this study, a total of $32.5 \%$ of the identified SMM manifested as abscess-like intramuscular lesions. However, some authors like Lenchik et al. [7] conclude that in the absence of clinical history, CT alone is not accurate for diagnosis and thus percutaneous aspiration is required. Similarly, Herring et al. [8] from the study of 15 patients with metastatic carcinoma to skeletal muscle and a review of 52 cases reported in the literature, was unable to find any clinical or radiographic characteristics that distinguish metastatic carcinoma to muscle from soft tissue sarcomas.

Our case is unusual for two reasons: the unusual spread of metastasis and the long latency period from initial therapy. There are only a few cases in the literature reporting abscess-like metastasis from cervical carcinoma $[5,9,10]$. Kalra et al. [11] reported a similar case involving a 60 year-old woman who discloses an abscess-like metastasis from cervix carcinoma two years after radiotherapy. In this case, fine needle aspiration cytology was realized two times to make an accurate diagnosis. This long latency period can be explained by late development of para-aortic or supraclavicular node metastasis after the initial treatment and the large volume represented by retroperitoneal space [12]. Thus, Narten et al. [13] reported a case of a 66- year-old white woman which remained relatively asymptomatic for 26 years after radiation therapy for stage III squamous cell cervix carcinoma in which the diagnosis was made only at necropsy which revealed a large retroperitoneal mass. Fine needle and trocard biopsy under radiologic guidance can be proposed as first diagnostic modality for such patients as in our case the aspirate from the left psoas show the presence of tumor cells. It may obviate the need for surgery in some cases $[2,3,5,10]$.

The prognosis is poor because IPM is related to systemic spread and discourages the use of an aggressive local therapy $[4,8,10,11]$ Radiotherapy is effective in the local and regional treatment of cervical carcinoma but would not have been useful for the extensive intraabdominal tumor deposits in the psoas muscle. However, it provided effective palliation of pain.

This case highlight the fact that metastasis from squamous cell carcinoma of the cervix can present as a psoas abscess clinically and radiologically and demonstrates certain difficulties in diagnosis even with the newer imaging modalities.

\section{Acknowledgements}

The authors would like to thank Dr. Lezrek Mohamed for her invaluable help in preparing this case report.

\section{References}

1. Singh AK, Gervais DA, Hahn PF, Mueller PR (2008) Neoplastic iliopsoas masses in oncology patients: CT findings. Abdom Imaging 33: 493-497.

2. Yang WT, Yeo W, Metreweli C (1999) Imaging of iliopsoas metastasis. Clin Radiol 54: 85-89.

3. Koike Y, Hatori M, Kokubun S (2005) Skeletal muscle metastasis secondary to cancer--a report of seven cases. Ups J Med Sci 110: 75-83.

4. Surov A, Hainz M, Holzhausen HJ, Arnold D, Katzer M, et al. (2010) Skeleta muscle metastases: primary tumours, prevalence, and radiological features. Eur Radiol 20: 649-658.

5. Devendra K, Tay SK (2003) Metastatic carcinoma of the cervix presenting as a psoas abscess in an HIV-negative woman. Singapore Med J 44: 302-303.

6. Pretorius ES, Fishman EK (2000) Helical CT of skeletal muscle metastases from primary carcinomas. AJR Am J Roentgenol 174: 401-404.

7. Lenchik L, Dovgan DJ, Kier R (1994) CT of the iliopsoas compartment: value in differentiating tumor, abscess, and hematoma. AJR Am J Roentgenol 162: 83-86.

8. Herring CL Jr, Harrelson JM, Scully SP (1998) Metastatic carcinoma to skeleta muscle. A report of 15 patients. Clin Orthop Relat Res 272-281.

9. Bar-Dayan Y, Fishman A, Levi Z, Rachmani R (1997) Squamous cell carcinoma of the cervix with psoas abscess-like metastasis in an HIV-negative patient. Isr J Med Sci 33: 674-676.

10. George J, Lai FM (1995) Metastatic cervical carcinoma presenting as psoas abscess and osteoblastic and lytic bony metastases. Singapore Med J 36: 224227 .

11. Kalra N, Aiyappan S, Nijhawan R, Sharma SC, Khandelwal N (2009) Metastatic carcinoma of cervix mimicking psoas abscess on imaging: a case report. J Gynecol Oncol 20: 129-131.

12. Sakurai H, Mitsuhashi N, Takahashi M, Akimoto T, Muramatsu H, et al. (2001) Analysis of recurrence of squamous cell carcinoma of the uterine cervix after definitive radiation therapy alone: patterns of recurrence, latent periods, and prognosis. Int J Radiat Oncol Biol Phys 50: 1136-1144.

13. Narten NC, Wettach GE, Neurnberger SP (1977) Difficulties in diagnosing unusual spread of cervical carcinoma. South Med J 70: 124-125. 